УДК 621.9

ВАК 05.02.07

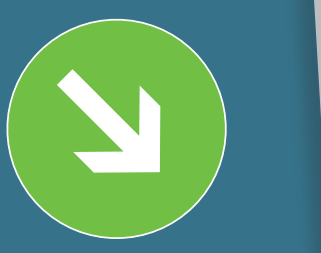

Ключевые слова: уровень локализации, конкурентоспособность, технологическая независимость и безопасность, технологическая трудоемкость

\title{
ЭФФЕКТИВНОСТЬ ЛОКАЛИЗАЦИИ \\ В СТАНКОСТРОЕНИИ
}

\author{
Александр КУзНЕЦОВ
}

\begin{abstract}
Рассмотрены различные аспекты и методология оценки уровня локализации производства станкостроительной продукции и высокотехнологичных компонент как с технологической, так и с экономической точки зрения. Показана связь нормирования уровня локализации производства станков и изделий общестанкостроительного применения для достижения технического уровня, обеспечивающего технологическую независимость и безопасность.
\end{abstract}

Вопрос эффективности локализации для таких высокотехнологичных производств, как станкостроение, остается дискуссионным. Прежде всего потому, что доля добавленной стоимости, которая локализуется в рамках проектов таких производств, может быть невелика. Разумеется, в наукоемкой станкостроительной отрасли промышленности, помимо текущей коммерческой эффективности, следует учитывать и стратегические соображения технологической независимости и безопасности страны. С этой точки зрения локализация производства станкостроительной продукции и высокотехнологичных компонент, особенно для критически значимых деталей, узлов и механизмов станков [1], а также станков и технологических комплексов с применением так называемых критических технологий [2] - важное направление ликвидации технологического отставания, сложившегося в станкоинструментальной отрасли промышленности.

Из-за технологических особенностей проектирования и производства станков существует риск того, что локализованные в стране производства не смогут стать конкурентоспособными в долгосрочной перспективе, если будут работать только на внутренний рынок. Необходимо обеспечивать сбыт станков и комплектующих на мировых рынках. Однако остается вопрос - насколько такая интеграция в мировую будет эффективной, как с технической, так и с коммерческой точки зрения.

В связи с этим реализация на практике Постановления Правительства РФ № 239 от 7 марта 2019 года «Об установлении запрета на допуск отдельных видов товаров станкоинструментальной промышленности...» требует решения задач локализации или организации станкоинструментальной продукции с наибольшей эффективностью, тем более критически значимой номенклатуры.

Для этого Минпромторгом России создается реестр российской станкоинструментальной продукции, основанием для включения в который служит Постановление Правительства РФ № 719 от 17 июля 2015 года «О критериях отнесения промышленной продукции к промышленной продукции, не имеющей аналогов, произведенных в Российской Федерации».

Методологическим вопросам и методам определения аналогов посвящена работа [3], в которой даны определения аналогов и предложена система их выбора из базы данных оборудования, а в [4] приведены методологические вопросы количественной оценки степени локализации, методы оценки и критерии уровня локализации станков.

В машиностроении 80-85\% затрат предопределяются техническими решениями, которые формируются в процессе конструирования и разработки технологии. На определенных этапах развития создаются условия для эволюционных скачков, связанных с накоплением научно-технического потенциала в обществе. К таким скачкам в развитии металлорежущих станков следует отнести появление станков с ЧПУ в 50-е годы XX века (первый скачок) и переход к модульному принципу проектирования и производства станков с ЧПУ на базе мехатронных модулей и устройств (второй скачок) - отличительной особенностью современного этапа развития станков. 
Область требований к технологическому оборудованию и металлорежущим станкам в частности содержит и определяет параметры, характеристики и свойства объекта рассмотрения и его структурных частей, которые обусловлены требованиями промышленности. Тогда при проектировании новых станков, разработке конструкторской и технологической документации требуется определить наилучшие из возможно достижимых параметров, характеристик и свойств объекта проектирования. Таким образом, в настоящее время похожие станки по своему технологическому назначению могут отличаться и отличаются по своему технико-экономическому уровню, который определяется используемой областью знаний о процессах и явлениях, содержанием и методами описания поведения, состояния, структуры и свойств создаваемого станка. Это обстоятельство также привело к снижению в 5-7 и более раз количества «оригинальных» деталей станков, а общий объем деталей и узлов за последние 30 лет снизился с тысяч и десятков тысяч единиц до сотен и тысяч деталей и узлов, при одновременном росте уровня их наукоемкости.

Станки являются важным конкурентным преимуществом для любой национальной промышленности. Для создания новых, более прогрессивных и инновационных станков необходимо не только обеспечить структурные (жесткость, пространство обработки и т.п.) и функциональные возможности (функции формообразования, управления, контроля и т.п.), основанные на новых принципах проектирования станка, но и быстро внедрять передовые технологии, материалы и компоненты, разрабатывать новые технологии в области станкостроения.

Эти процессы, в свою очередь, привели к изменению структуры производства данного вида оборудования. Произошла трансформация станкостроительных заводов из предприятий полного технологического цикла в относительно компактные, тяготеющие к сборочному производству предприятия с подразделениями финишной механообработки и обработки критически значимых и оригинальных деталей и узлов. Изготовление наукоемких комплектующих общестанкостроительного применения сосредоточилось среди достаточно ограниченного числа крупных производителей, способных обеспечить их серийное производство и приемлемую стоимость.

Рассмотрим обобщенную типовую схему сборки станка из его элементов (рис. 1) как результат реализации и воплощения в материальном объекте всех воздействий: от проектирования и разработки конструкторской и технологической документации до преобразования и производства замысла в станок. Базовый перечень технологических процессов, обеспечивающих производство станков приведен в табл.1.

Такое представление позволяет формировать различные виды производств как по уровню охва-

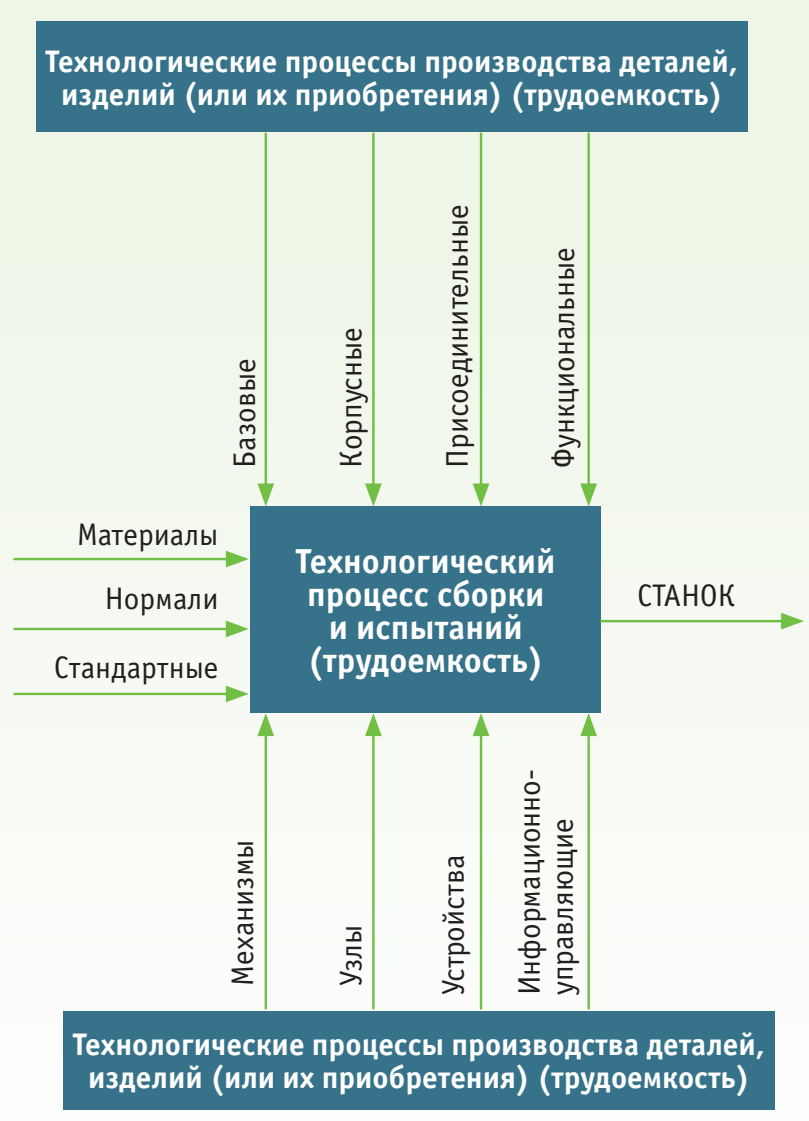

Puc. 1. Типовая схема сборки станка из его элементов

та полного цикла производства станка в целом, так и на уровне кооперации, обусловленной показателями технологической и экономической эффектив-

\section{Таблица 1. Виды технологических процессов производства элементов станков}

\begin{tabular}{|l|l|}
\hline Код & $\begin{array}{l}\text { Вид технологического процесса по методу выпол- } \\
\text { нения }\end{array}$ \\
\hline 01 & Операции общего назначения \\
\hline 02 & Технический контроль \\
\hline 03 & Перемещение \\
\hline 04 & Испытания \\
\hline 05 & Обработка давлением \\
\hline 06 & Обработка резанием \\
\hline 07 & Термообработка \\
\hline 08 & Получение покрытий (металлических и неметалли- \\
\hline 10 & Песких неорганических) \\
\hline 11 & Электромонтаж \\
\hline 12 & Сборка \\
\hline 13 & Сварка \\
\hline
\end{tabular}


ности производства, а также рядом социальных и иных вопросов. Все это обусловливает необходимость решения задачи оптимального (рационального) уровня локализации производства изделий той совокупностью их элементов, которая определяет технологическую независимость страны.

Сравнение методов оценки эффективности уровня локализации базируется на том, насколько полно они дают ответы на ряд важных вопросов:

$\rightarrow$ полнота и достоверность конструкторской и технологической документации;

$\rightarrow$ оценка технико-технологического уровня и конкурентоспособности в сравнении со «станкоманалогом» и прогнозом направлений развития;

$\rightarrow$ возможность, целесообразность и эффективность создания «сборочного производства» и вид организации его производства (крупноузловая сборка, узловая сборка и т.п.);

$\rightarrow$ возможность, целесообразность и эффективность создания «локальных производств» разной степени уровня локализации и вид организации этих производств (сборочное с собственным производством критических элементов и т.п.);

$\rightarrow$ эффективность создания и организации «производства полного цикла».

Критически значимые составляющие обязательны для оценки уровня локализации, доля которых не ниже заданного уровня локализации. Критически значимые элементы станка служат основанием для обеспечения технологической независимости и технологической безопасности изделия для России $[1,2]$.

Рассмотрим методы оценки уровня локализации производства, на базе которых можно было бы ответить на ряд вопросов о состоянии, целесообразности, технологической и экономической эффективности того или иного локального производства.

\section{ОЦЕНКА УРОВНЯ ЛОКАЛИЗАЦИИ ПО КОЛИЧЕСТВУ ЭЛЕМЕНТОВ СТАНКА}

Предположим, что нами сформировано множество элементов станка, состоящее из $N$ наименований (А, Б, С, Д...), из которых $N_{p}(\mathrm{~A}, \mathrm{E}$, К...) производятся на предприятии или по кооперации внутри страны. Очевидно, что $N_{p}<N$, причем выполняется равенство $N=N_{p}+N_{i}$, где $N_{i}$ будем называть множеством импортируемых элементов станка. Импортная деталь определяется как изделие, изготовленное не на территории России. Объединение нескольких импортных деталей (двух и более) с целью получения нового изделия с новыми свойствами, параметрами и характеристиками является условно произведенным в России, то есть так называемой российской импортонезамещенной продукцией.

Тогда зависимость для оценки уровня локализации может быть записана следующим образом [4]:
$U_{N}=\frac{N_{p}}{N}=\frac{N_{p}}{N_{p}+N_{i}}=\frac{1}{1+\frac{N_{i}}{N_{p}}}=\frac{N_{p}+N_{i}-N_{i}}{N_{p}+N_{i}}=1-\frac{N_{i}}{N_{p}+N_{i}}=1-\frac{1}{1+\frac{N_{p}}{N_{i}} \mathrm{a}},(1)$

значение этой величины находится в диапазоне от 0 до 1 и по своей сути определяет долю импортных частей в станке или соотношение импортных и отечественных элементов станка $-\frac{N_{i}}{N_{p}}$, изменяющееся от 0

до $N$. Поэтому, по сути, выражение (1) определяет долю российских $\frac{N_{p}}{N_{p}+N_{i}}$ или импортных $\frac{N_{i}}{N_{p}+N_{i}}$ деталей

в станке. Для подсчета параметра локализации важно определиться с объемом рассматриваемого количества элементов станка $N=N_{p}+N_{i}$ или, иначе говоря, принципа деления станка на элементы. В настоящее время опубликованы два варианта этого подхода соответственно в Постановлении Правительства РФ № 719 от 17 июля 2015 года и в [1, 4]. Кроме того, в [1] показано, что, исходя из рекомендаций Постановления № 719, если суммарное количество элементов, составляющих станок $N=42$, то для достижения предельного уровня локализации в 70\% количество элементов российского производства должно составлять не более 30 элементов. Исходя из приведенного перечня, для достижения этого уровня можно не производить, например, 12 следующих элементов станка:

$\rightarrow$ поперечины, ползуны, кривошипы, шатуны, станина, портал, колонны и т.п.;

$\rightarrow$ мотор-шпиндель;

$\rightarrow$ накладной стол - плоский (устанавливаемый на суппорте), в том числе для трехкоординатной обработки;

$\rightarrow$ направляющие качения, скольжения и др.;

$\rightarrow$ винтовые передачи роликовые, шариковые и др.;

$\rightarrow$ стойки и др. менее ответственные корпусные, габаритные детали;

$\rightarrow$ средние и мелкие корпусные детали;

$\rightarrow$ коробки передач;

$\rightarrow$ управляющий программно-аппаратный комплекс;

$\rightarrow$ лазерные указатели, датчики положения бесконтактные и др.;

$\rightarrow$ опоры, в том числе прецизионные подшипники качения, опоры скольжения, гидро-, аэро-, магнитные опоры и др.;

$\rightarrow$ одноосевой стол.

Следовательно, на основании принятого допущения о количестве элементов и способе их выбора можно получить локализованный станок с уровнем в 70\%, для которого не производится ни один элемент, составляющий основу и суть самого понятия станок. Таким образом, такой методический подход не приводит к развитию станкостроения, а является лишь имитацией развития, если не сказать, тормозом, приводит к потере знаний и опыта в области станкостроения. 
Принципиально возможным методом оценки, как предельный случай, можно считать метод, когда количество элементов станка $N=N_{p}+N_{i}$ определяется по ведомостям ГОСТ 2.102-2013 «Виды и комплектность конструкторских документов» и ГОСТ 2.106-96 «Единая система конструкторской документации. Текстовые документы». В этом случае общее количество элементов станка $N$ в терминах ГОСТ 2.101-2016 «Виды изделий» достаточно велико, но является абсолютно полным, что обусловит, с одной стороны, достаточно большую трудоемкость проведения расчетов уровня локализации, а с другой - определит и обеспечит высокую их точность.

Возможны и иные варианты формирования базы количества элементов станка $N=N_{p}+N_{i}$ для оценки уровня локализации, например, на основе сборочного чертежа, чертежей деталей, ведомостей спецификаций и покупных изделий. В этом случае обязательным является разработка принципа классификации групп узлов и деталей для отнесения к ним этих элементов станка. Один из возможных принципов такой классификации приведен в [1, 4].

Например, перечень классификационных групп может быть принят следующим:

$\rightarrow$ базовые и корпусные детали изделия (товара);

$\rightarrow$ детали узлов и механизмов изделия (товара);

$\rightarrow$ узлы и механизмы общестанкостроительного применения;

$\rightarrow$ системы и устройства информационные, мехатронные, адаптронные, электроавтоматики и т.п.;

$\rightarrow$ устройства и системы технологического обеспечения;

$\rightarrow$ стандартные изделия и нормали;

$\rightarrow$ прочие детали, механизмы, узлы, устройства, аппараты, системы, в том числе общемашиностроительного применения.

Следует сделать одно замечание об отнесении элемента станка к понятию «произведено в России». Запишем количество элементов в станке в виде:

$$
N=N_{p}+N_{p i}+N_{i}
$$

где $N_{p i}$ - является так называемой российской импортонезамещенной или условно российской (объединение нескольких импортных деталей (двух и более) с целью получения нового изделия с новыми свойствами, параметрами и характеристиками является условно произведенным в России) продукцией. Очевидно, исходя из этого определения, что станок, собранный на территории РФ из импортных узлов, комплектов, агрегатов и устройств также является $\underset{N_{p} \rightarrow 0}{\mathrm{yc}} \mathrm{\text {уовно }}$ российским, так как $U_{N}=\frac{N_{p} \rightarrow 0}{\left(N_{p} \rightarrow 0\right)+\left(N_{p i} \rightarrow 0\right)+N_{i}} \approx 0$. Это следует из выражения

(1), если записать его в следующем виде:

$$
U_{N}=\frac{N_{p}}{N}=\frac{N_{p}}{N_{p}+N_{p i}+N_{i}} \neq \frac{N_{p}+N_{p i}}{N_{p}+N_{p i}+N_{i}} .
$$

Элементы станка типа $N_{p i}$ можно признать условно российскими, если доля российских элементов в таком изделии не меньше требуемого уровня локализации, который определяется по выражению (1), то есть этот элемент рассматривается как самостоятельное изделие. Например, стружкотранспортер состоит из корпуса, ленты, двигателя, муфты, редуктора. При этом уровень требуемой локализации станка равен 0,5. Кроме ленты и корпуса все детали импортные. Тогда $U_{p i}=2: 5=0,4$, следовательно, этот элемент станка не относится к российскому производству $N_{p}$.

Таким образом, оценка уровня локализации по зависимости (1) позволяет с большей или меньшей степенью точности охарактеризовать уровень «импортопотребления» или оценить только соотношение долей элементов станка российского и импортного производств как относительно друг друга, так и в иных соотношениях из набора элементов станка в соответствии с принятым принципом их классификационного отнесения. Ответить на вопросы целесообразности, рациональности, эффективности технологической или финансовой, вида и серийности производства станков, способа его организации (сборка из узлов и агрегатов, производства деталей по кооперации или самим или в каком соотношении и т.п.) не представляется возможным.

\section{ОЦЕНКА УРОВНЯ ЛОКАЛИЗАЦИИ ПО ТЕХНОЛОГИЧЕСКОЙ ТРУДОЕМКОСТИ СОЗДАНИЯ СТАНКА}

Изложенное выше, по нашему мнению, недостаточно строго и однозначно определяет уровень импортозамещения исходя из того, что, например, система (как объект производства: станок, робот, технологическое оборудование и т.п.) может состоять и включать только те детали и узлы, механизмы и устройства собственного производства, которые в общем объеме трудоемкости изготовления занимают незначительную часть, хотя количество таких деталей и узлов велико.

Поясним сказанное на простом примере. Допустим, что для создания станка необходимо $N$ деталей и узлов и включает $N_{i}$ импортных деталей, узлов и $N_{p}$ - произведенных на территории России. На производство $i$-й детали требуется $t_{i}$ единиц времени. Примем, для простоты вычислений, что станок состоит из $N=5$ деталей и узлов, причем количество импортных деталей и узлов $N_{i}=2$. На производство $i$-й детали и узла затрачивается соответственно: $t_{1}=2$, $t_{2}=5, t_{3}=8, t_{4}=1, t_{5}=4$ единиц времени и требуется $t_{a}=6$ единиц времени для сборки станка. Предположим, что станки состоят из деталей и узлов содержащих следующие элементы: 
$\rightarrow \quad$ I вариант: $i=1,2,3 ; N_{p}=3 ; t_{1}=2 ; t_{2}=5 ; t_{3}=8$;

$\rightarrow$ II вариант: $i=1,4,5 ; N_{p}=3 ; t_{1}=2 ; t_{4}=1 ; t_{5}=4$;

$\rightarrow$ III вариант: $i=2,4,5 ; N_{p}=3 ; t_{2}=5 ; t_{4}=1 ; t_{5}=4$. Определим уровень локализации (табл. 2) для указанных вариантов по следующим зависимостям:

$$
\begin{gathered}
U_{N}=N_{p} / N ; \\
U_{\mathrm{T}}=\sum_{i=1}^{N_{p}} t_{i} / \sum_{i=1}^{N} t_{i} ; \\
U_{\mathrm{Ta}}=\left(\sum_{i=1}^{N_{p}} t_{i}+t_{\mathrm{a}}\right) /\left(\sum_{i=1}^{N} t_{i}+t_{\mathrm{a}}\right) .
\end{gathered}
$$

Таблица 2. Варианты оценок уровня локализации

\begin{tabular}{|l|l|l|l|l|l|l|l|l|l|l|}
\hline Вариант & $t_{1}$ & $t_{2}$ & $t_{3}$ & $t_{4}$ & $t_{5}$ & $t_{a}$ & $U_{N}$ & $U_{T}$ & $U_{T a}$ & $U_{U}$ \\
\hline $1-N_{p}$ & 2 & 5 & 8 & - & - & 6 & 0,6 & 0,75 & 0,8 & 0,48 \\
\hline $2-N_{p}$ & 2 & - & - & 1 & 4 & 6 & 0,6 & 0,35 & 0,5 & 0,3 \\
\hline $3-N_{p}$ & - & 5 & - & 1 & 4 & 6 & 0,6 & 0,5 & 0,62 & 0,37 \\
\hline $4-N_{p}$ & - & - & - & - & - & 6 & 0 & 0 & 0,23 & 0 \\
\hline
\end{tabular}

Как видно (табл. 2) из сравнения полученных величин значений $U_{N}, U_{\mathrm{T}}, U_{\mathrm{Ta}}$, найденное по (1) значение $U_{N}$ одинаково для всех трех вариантов производства станка, хотя в каждом варианте производства имеются различные $i$-е детали российского производства с разной трудоемкостью изготовления. Поэтому если оценивать уровень импортоприменения (импортозамещения) по (2), то явно видно, что не равнозначные по существу производства станков становятся равноценными. Подобный факт приводит к корректной оценке уровня импортоприменения (импортозамещения) для такой производственной системы.

Следовательно, показатель уровня импортоприменения (импортозамещения), найденный по (1), (2), не может служить объективной оценкой уровня локализации. На самом деле, как это следует из приведенного примера и анализа выражений $(1,2)$, он характеризует степень (полноту) охвата деталей и узлов отечественного производства и говорит лишь о том, какая доля элементов станка подлежит импортозамещению.

Второй показатель $U_{\mathrm{T}}$, найденный для трех вариантов системы производства станка, оказался неодинаковым, и его значение колеблется в широких пределах: от 35\% для второго варианта до 75\% для первого варианта. Этот показатель характеризует величину уровня технологической импортозависимости (технологической локализации) производства всех составляющих элементов (деталей и узлов) станка (см. рис. 1), то есть является объективной характеристикой, дающей представление об эффектив- ности в достижении поставленной цели - снижение уровня импортозависимости (импортопотребления).

Третий показатель $U_{\text {Ta }}$ для трех вариантов системы производства станка оказался также неодинаковым и отличным от $U_{N}$ и $U_{\mathrm{T}}$, а его значение колеблется в пределах: от 50\% для второго варианта до $80 \%$ для первого варианта. Этот показатель характеризует величину уровня производственно-технологической импортозависимости (производственно-технологической локализации) полного цикла производства, включающего производство всех составляющих элементов (деталей и узлов) станка и его сборки и испытаний (см. рис. 1), т.е. является объективной характеристикой, дающей представление о производственнотехнологической эффективности в достижении поставленной цели - степени снижения уровня импортозависимости (импортопотребления).

Интегральный показатель локализации $U_{U}=U_{N} U_{\mathrm{Ta}}$ характеризует фактический уровень производственной локализации станка, а его значение колеблется в пределах: от 30\% для второго варианта до 48\% для первого варианта, что, как сопоставимый ряд, корреспондируется между всеми показателями, значения которых оценивают и определяют различную глубину свойства «локализация» производства станков, однако без стоимостных составляющих не говорят об эффективности такого производства.

Определим в общем виде указанные показатели, учитывая, что для любой системы производства станков (см. рис. 1), в соответствии с ее организационной структурой, суммарные затраты времени $T$ производства $N$ элементов станка состоят из: $T_{\mathrm{p}}$ (индекс «Р» обозначает полностью произведено в России), связанного с изготовлением деталей на собственном (отечественных предприятиях) производстве; затрат времени $T_{p u}$, связанных с изготовлением узлов, механизмов устройств на собственном (отечественных предприятиях) производстве из импортных деталей, а также приведенных затрат $T_{u}$, связанных с применяемыми импортными изделиями и в процессах сборки станка, затраты на которую составляют $T_{a}$. Соотношения указанных составляющих затрат времени определяют различные свойства производственной системы: степень или уровень применяемости, локализации, полноты охвата системы отечественными деталями и др. Действительно, если доля импортных изделий велика по сравнению с отечественными, то такая система зависима от внешних факторов и в этом смысле малоэффективна и является технологически зависимой, так как уровень локализации производства ее компонентов низкий.

Тогда показатель производственно-технологической локализации - уровень импортоприменения (импортозамещения) - будет равен: 
$U_{\mathrm{Ta}}=\frac{\sum_{i=1}^{N_{p}} T_{p i}+T_{a}}{\sum_{i=1}^{N_{p}} T_{p i}+T_{a}+\sum_{j=1}^{N_{p u}} T_{p u j}+\sum_{k=1}^{N_{u}} T_{u k}}=1-\frac{\sum_{j=1}^{N_{p u}} T_{p u j}+\sum_{k=1}^{N_{u}} T_{u k}}{\sum_{i=1}^{N_{p}} T_{p i}+T_{a}+\sum_{j=1}^{N_{p u}} T_{p u j}+\sum_{k=1}^{N_{u}} T_{u k}}=$

$=1-\frac{1}{\frac{\sum_{i=1}^{N_{p}} T_{p i}+T_{a}}{\sum_{j=1}^{N_{p u}} T_{p u j}+\sum_{k=1}^{N_{u}} T_{u k}}+1}=1-\frac{1}{1+U_{p}}=\frac{U_{p}}{1+U_{p}}$,

где $U_{p}=\frac{\sum_{i=1}^{N_{p}} T_{p i}+T_{a}}{\sum_{j=1}^{N_{p u}} T_{p u j} \sum_{k=1}^{N_{u}} T_{u k}}-$ степень локализации собствен-

Преобразуем выражения для оценки $U_{p}$ к виду, в соответствии с методом оценок работы [6]:

$U_{p}=\frac{\sum_{i=1}^{N_{p}} T_{p i}+T_{a}}{\sum_{j=1}^{N_{p u}} T_{p u j}+\sum_{k=1}^{N_{u}} T_{u k}}=\frac{T_{a}}{\sum_{j=1}^{N_{p u}} T_{p u j}} \cdot \frac{\sum_{i=1}^{N_{p}} T_{p i}+T_{a}}{T_{a}} \cdot \frac{\sum_{j=1}^{N_{p u}} T_{p u j}}{\sum_{j=1}^{N_{p u}} T_{p u j}+\sum_{k=1}^{N_{u}} T_{u k}}=$

$=T_{a} \cdot \frac{1+\frac{\sum_{i=1}^{N_{p}} T_{p i}}{T_{a}}}{\sum_{j=1}^{N_{p u}} T_{p u j}+\sum_{k=1}^{N_{u}} T_{u k}}$,

где:

$U_{p a}=\frac{\sum_{1}^{N_{p}} T_{p i}}{T_{a}}-$ уровень технологической сложности про-

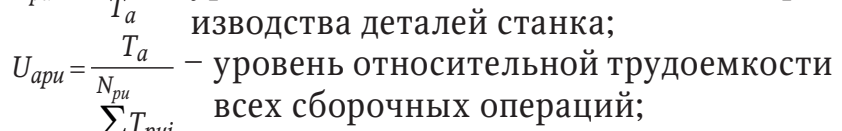
$\sum_{j=1}^{N_{p u}} T_{p u j} \quad$ всех сборочных операций;

$U_{p u}=\frac{\sum_{j=1}^{N_{p u}} T_{p u j}}{N_{p u}}-$ уровень относительного импор$\sum_{p u}=\frac{N_{j u}}{\sum_{j=1}^{N_{p u j}} T_{k=1} T_{u k}}$ тозамещения;

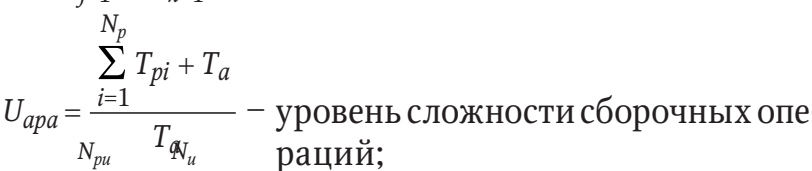
$U_{a u}=\frac{\sum_{j=1}^{N_{p u}} T_{p u j}+\sum_{k=1} T_{\mathbb{Q}_{u}}}{T_{a}}-$ раций; Тогда (5) запишется в виде:

$$
U_{p}=U_{a p u} U_{a p a} U_{p u}=U_{a u}^{-1}(1+U p a) .
$$

Если $T a=0$, то есть сборочные операции не рассматриваются, то показатель технологической локализации будет равен:

$$
\begin{aligned}
& U_{T}=\frac{\sum_{i=1}^{N_{p}} T_{p i}}{\sum_{i=1}^{N_{p}} T_{p i}+\sum_{j=1}^{N_{p u}} T_{p u j}+\sum_{k=1}^{N_{u}} T_{u k}}=1-\frac{\sum_{j=1}^{N_{p u}} T_{p u j}+\sum_{k=1}^{N_{u}} T_{u k}}{\sum_{i=1}^{N_{p}} T_{p i}+\sum_{j=1}^{N_{p u}} T_{p u j}+\sum_{k=1}^{N_{u}} T_{u k}}= \\
& =1-\frac{1}{1+U_{T p}}=\frac{U_{T p}}{1+U_{T p}},
\end{aligned}
$$

где $U_{T p}=\frac{\sum_{i=1}^{N_{p}} T_{p i}}{\sum_{j=1}^{N_{p u}} T_{p u j}+\sum_{k=1}^{N_{u}} T_{u k}} \begin{aligned} & \text { степень технологической лока- } \\ & \text { лизаци собственного произ- } \\ & \text { водста. }\end{aligned}$

И если $T_{p}=0$, то есть нет собственного производства элементов станка, то получим показатель уровня локализации сборочного производства:

$$
U_{\text {лa }}=\frac{T_{a}}{T_{a}+\sum_{j=1}^{N_{p u}} T_{p u j}+\sum_{k=1}^{N_{u}} T_{u k}} .
$$

Запишем (3) в следующем виде:
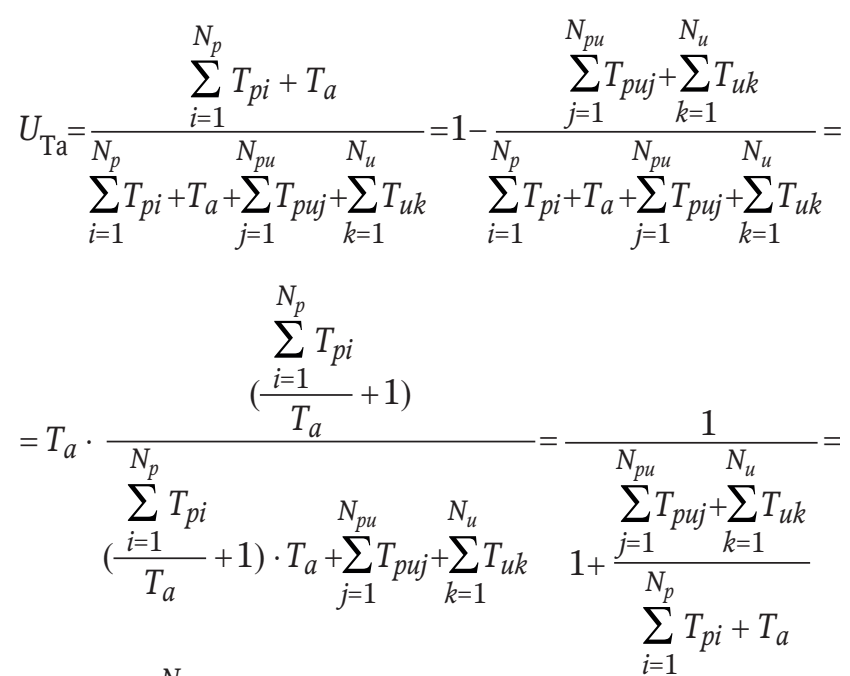

$$
=\frac{\frac{\sum_{i=1}^{N_{p}} T_{p i}}{T_{a}}+1}{1+\frac{\sum_{i=1}^{N_{p}} T_{p i}+\sum_{j=1}^{N_{p u}} T_{p u j}+\sum_{k=1}^{N_{u}} T_{u k}}{T_{a}}}=\frac{1}{\sum_{i=1}^{N_{p}} T_{p i}+T_{a}}=1-\frac{1}{1+U_{p}}=
$$

$=\frac{U_{p}}{1+U_{p}}=\frac{U_{a p u} \cdot U_{a p a} \cdot U_{p u}}{1+U_{a p u} \cdot U_{a p a} \cdot U_{p u}}$. 
Тогда получим следующие показатели:

$U_{p u}=\frac{\sum_{1}^{N_{p u}} T_{p u j}+\sum_{1}^{N_{u}} T_{u i}}{\sum_{1}^{N_{p}} T_{p i}+T_{a}}$ уровень технологической сложности производства импортных деталей станка или уровень технологической зависимости;

$U_{\text {сла }}=\frac{\sum_{i=1}^{N_{p}} T_{p i}+\sum_{j=1}^{N_{p u}} T_{p u j}+\sum_{k=1}^{N_{u}} T_{u k}}{T_{a}}-$

уровень технологической сложности сборки станка.

Таким образом, полученная совокупность показателей служит методической основой для анализа уровня технологической локализации и ее целесообразности.

Для оценки уровня локализации с экономической (финансовой) точки зрения необходимо рассмотреть затраты на производство в соответствии с его производственной и технологической организацией (рис. 1) при производстве станков. Для всех предприятий, вне зависимости от организационноправовых форм, установлен единый перечень экономических элементов затрат, отражающих себестоимость продукции. В табл. 3, в соответствии с [7, 8], приведен состав затрат и их классификация. Таким образом, в сумму затрат по всем вышеперечисленным элементам входят затраты на производство запланированного объема продукции:

$$
\begin{aligned}
3_{\text {пр }}=3_{\text {с }}+3_{\text {м }}+3_{\text {пр }}+3_{\text {вм }}+3_{\text {т }}+3_{\text {эн }}+3_{\text {п }}+3_{\text {от }}+\mathrm{A}+ \\
+3_{\text {рсп }}+3_{\text {др }}=\sum 3_{\mathrm{i}}+\mathrm{A}
\end{aligned}
$$

где $3_{\text {пр }}$ - затраты на производство; $3_{c}$ - стоимость сырья; 3 м - стоимость основного материала за вычетом возвратных отходов; $3_{\text {пр }}$ - стоимость покупных комплектующих изделий и полуфабрикатов; $3_{\text {вм }}$ - стоимость вспомогательных материалов; $3_{\text {т }}$ - стоимость топлива; $3_{\text {эн }}$ - стоимость энергии; $3_{\text {п }}$ - заработная плата; $3_{\text {от }}$ - отчисления на социальное страхование; А - амортизация основного капитала; $3_{\text {рсп }}$ - затраты по обеспечению работоспособности основного капитала; $3_{\text {др }}$ - прочие денежные расходы.

Экономическая сущность расчета точки безубыточности состоит в анализе взаимодействия спроса и предложения по конкретному товару предприятия. При этом надо учитывать, что своим предложением предприятие может управлять, так как его формируют затраты предприятия, а спросом управлять нельзя, можно лишь в некоторой степени повлиять на него. В процессе такого анализа определяется точка безубыточности, соответствующая объему выпускаемой продукции при заданном (анализируемом) уровне цен, при кото- ром доход от продажи равен издержкам производства. Точка безубыточности определяется как отношение постоянных издержек производства к разнице между ценой и удельными переменными издержками. Можно также рассчитать и минимальную цену, по которой реализуется продукция исходя из заданного объема продаж, суммы постоянных и удельных переменных издержек производства. Себестоимость продукции, работ, услуг представляет собой стоимостную оценку используемых в процессе производства продукции (работ, услуг) природных ресурсов, сырья, материалов, топлива, энергии, основных фондов, трудовых ресурсов, а также других затрат на ее производство и реализацию.

Итоговое рассмотрение классификации затрат дает представление об определенной зависимости общей суммы затрат и себестоимости единицы продукции от объема производства. Себестоимость может быть представлена в виде:

$$
C=\frac{C_{a}+C_{b} \cdot K}{K},
$$

где $C_{a}$ - абсолютная сумма постоянных расходов; $C_{b}$ - переменные расходы на единицу продукции (услуг); $K$ - объем производства продукции.

Тогда выражение (3) в единицах стоимости запишется:

$$
U_{e}=\frac{\sum_{i=1}^{N_{p}}\left(\frac{C_{a}+C_{b} \cdot K}{K}\right)_{p i}+\left(\frac{C_{a}+C_{b} \cdot K}{K}\right)_{a}}{\sum_{i=1}^{N_{p}}\left(\frac{C_{a}+C_{b} \cdot K}{K}\right)_{p i}+\left(\frac{C_{a}+C_{b} \cdot K}{K}\right)_{a}+\sum_{j=1}^{N_{p u}}\left(\frac{C_{a}+C_{b} \cdot K}{K}\right)_{p u j}+\sum_{k=1}^{N_{u}}\left(\frac{C_{a}+C_{b} \cdot K}{K}\right)_{u k}} \cdot
$$

Другие показатели уровня локализации выражений (3)-(9) структурно будут аналогичными и определяться путем замены в указанных выражениях соответствующих значений трудоемкости на себестоимость.

Очевидно, что в этом случае показатель уровня локализации, определенный по (10), не будет соответствовать аналогичному уровню, найденному по (3).

Запишем выражение (11) в следующем виде:

$$
\begin{aligned}
& U_{e}=\frac{\sum_{i=1}^{N_{p}}\left(\frac{C_{a}+C_{b} \cdot K}{K}\right)_{p i}+\left(\frac{C_{a}+C_{b} \cdot K}{K}\right)_{a}}{\sum_{i=1}^{N_{p}}\left(\frac{C_{a}+C_{b} \cdot K}{K}\right)_{p i}+\left(\frac{C_{a}+C_{b} \cdot K}{K}\right)_{a}+\sum_{j=1}^{N_{p u}}\left(\frac{C_{a}+C_{b} \cdot K}{K}\right)_{p u j}+\sum_{k=1}^{N_{u}}\left(\frac{C_{a}+C_{b} \cdot K}{K}\right)_{u k}}= \\
& =\frac{\sum_{i=1}^{N_{p}}\left(\frac{C_{a}+C_{b} \cdot K}{K}\right)_{p i}+\left(\frac{C_{a}+C_{b} \cdot K}{K}\right)_{a}}{S_{E W} \cdot V_{D D P}},
\end{aligned}
$$


Таблица 3. Состав затрат на производство станков

\begin{tabular}{|c|c|c|c|c|c|c|}
\hline \multirow[t]{2}{*}{ Статьи } & \multicolumn{2}{|c|}{ По составу затрат } & \multicolumn{2}{|c|}{$\begin{array}{l}\text { По способу отнесения на } \\
\text { себестоимость продукции }\end{array}$} & \multicolumn{2}{|c|}{$\begin{array}{c}\text { По объему производства } \\
\text { продукции }\end{array}$} \\
\hline & простые & $\begin{array}{c}\text { ком- } \\
\text { плексные }\end{array}$ & прямые & косвенные & $\begin{array}{l}\text { пропорци- } \\
\text { ональные }\end{array}$ & $\begin{array}{l}\text { непропорци- } \\
\text { ональные }\end{array}$ \\
\hline Сырье и материалы & + & & + & & + & \\
\hline $\begin{array}{l}\text { Покупные комплектующие изделия и полу- } \\
\text { фабрикаты }\end{array}$ & + & & + & & + & \\
\hline Возвратные отходы & + & & + & & + & \\
\hline $\begin{array}{l}\text { Топливо и энергия на технологические } \\
\text { цели }\end{array}$ & + & & + & & + & \\
\hline $\begin{array}{l}\text { Основная заработная плата производ- } \\
\text { ственных рабочих }\end{array}$ & + & & + & & + & \\
\hline Дополнительная заработная плата & + & & + & & + & \\
\hline Отчисления на социальное страхование & + & & + & & + & \\
\hline $\begin{array}{l}\text { Расходы на освоение и подготовку произ- } \\
\text { водства }\end{array}$ & & + & + & & + & \\
\hline Износ инструментов и приспособлений & & + & + & & + & \\
\hline $\begin{array}{l}\text { Расходы по эксплуатации и содержанию } \\
\text { оборудования }\end{array}$ & & + & & + & + & \\
\hline Цеховые расходы & & + & & + & & + \\
\hline Общезаводские расходы & & + & + & & & + \\
\hline Потери от брака & & + & + & & & + \\
\hline Прочие производственные расходы & & + & & + & & + \\
\hline Внепроизводственные расходы & & + & & + & & + \\
\hline
\end{tabular}

где $S_{E W}$ - цена аналога; $V_{D D P}$ - увеличение цены за счет дополнительных платежей и услуг (табл. 4).

Из (12) следует, что условием эффективности локализации является выражение:

$$
U_{e} \cdot S_{E W} \cdot V_{D D P} \geq \sum_{1}^{N_{p}}\left(\frac{C_{a}+C_{b} \cdot K}{K}\right)_{p i}+\left(\frac{C_{a}+C_{b} \cdot K}{K}\right)_{a},
$$

а условием эффективности сборочного производства является выражение:

$$
S_{E W} \cdot V_{D D P} \geq\left(\frac{C_{a}+C_{b} \cdot K}{K}\right)_{a} .
$$

При существующем в настоящее время состоянии производства изделий общестанкостроитель- ного применения, обеспечение нормативного уровня локализации в 50\% и более нередко еще до начала производства заранее предопределяет высокую стоимость станка по отношению к зарубежным аналогам (к стоимости изделий надо добавить стоимость доставки, НДС, пошлины и т.п.). Технический уровень станков также может отличаться не в лучшую сторону, так как часто применяются общестанкостроительные изделия более низкого технического уровня и стоимости (с целью оптимизации себестоимости) и, соответственно, недостаточного качества. Конструкции станков, а также технологическое обеспечение качества их производства либо в целом повторяет конструкцию зарубежных аналогов прежних лет (уже технически устарели), либо представляет собой доработанную (адаптированную) собствен- 
Таблица 4. К определению значений при оценке по выражениям (3-14)

\begin{tabular}{|c|c|c|c|c|c|}
\hline \multirow[t]{2}{*}{ Виды затрат } & \multicolumn{5}{|c|}{ Изделия, детали } \\
\hline & Базовые & Корпусные & Узлы & Стандартные & Станок \\
\hline Затраты & $+/-$ & $+/-$ & $+/-$ & $+/-$ & \\
\hline Трудоемкость изготовления & $+/-$ & $+/-$ & $+/-$ & $+/-$ & Сборка \\
\hline Транспорт* & $+/-$ & $+/-$ & $+/-$ & $+/-$ & $1-7 \%$ \\
\hline Таможенные налоги и сборы* & $+/-$ & $+/-$ & $+/-$ & $+/-$ & $\begin{array}{l}\text { НДС }(20 \%)+ \\
\text { Сбор }(0-10 \%)\end{array}$ \\
\hline Пусконаладочные работы* & & & & & $1-5 \%$ \\
\hline Стоимость склад завод-изготовитель* & & & & & ExWork \\
\hline
\end{tabular}

* Сведения приведены ориентировочно, относятся к импортируемой продукции и определяются соответствующими нормативными документами.

ную конструкцию под соответствующую комплектацию, что иногда приводит и к ее ухудшению, что обусловлено отсутствием системных исследований этих изменений конструкции. Следовательно, такая ситуация ведет не только к отставанию технологического уровня, но и к отставанию в направлениях и методах развития самих станков и их компонентной базы.

Только собственное производство критически значимых изделий, развитие их технического уровня, проведение исследований и работ по опережению конкурентных производителей обеспечит технологическую независимость.

Таким образом, комплексный показатель эффективности локализации производства станков заданного технического уровня и организационно-технологической системы производства может быть оценен как среднеарифметическое относительных показателей $U_{T e}=0,5\left(U_{U}+U_{e}\right)$. Анализ различных показателей эффективности локализации и оценка направления, выбор метода ее проведения на разных этапах определяется в зависимости от поставленной задачи и иных ограничений организационного, производственного и экономического характера на основании описанного в данной работе методического подхода.

\section{ЛИТЕРАТУРА}

1. Кузнецов А.П. От состояния локализации к развитию станкостроения // СТАНКОИНСТРУМЕНТ. 2018. № 4. С. 1-12.

2. Кузнецов А.П. Основные задачи формирования импортонезависимой станкоинструментальной отрасли в России // СТАНКОИНСТРУМЕНТ. 2016. № 2. С. $16-25$.

3. Кузнецов А.П. Технический уровень и конкурентоспособность металлорежущих станков // СТАНКОИНСТРУМЕНТ. 2016. № 4. С. 21-33.

4. Кузнецов А.П. Локализация в Российском станкостроении - фактор достижения технологической независимости. Методологические аспекты // СТАНКОИНСТРУМЕНТ. 2016. № 3. C. 41-54.

5. Бушуев В.В., Кузнецов А.П., Молодцов В.В. Особенности проектирования технологического оборудования на современном этапе развития // СТИН. 2015. № 8. С. 7-12.

6. Kuznetsov A.P., Koriath H.-J., Kalyashina A.V., Langer T. Equivalence Assessment Method for the Resource Efficiency of Equipment, Technologies and Production Systems. Procedia CIRP 15th Global Conference on Sustainable Manufacturing. Published by Elsevier B.V. 2018. V. 21. PP. 525-532. DOI: 10.1016/j. promfg.2018.02.153

7. Зайцев Н.Л. Экономика промышленного предприятия: Учебник / 4-е изд., перераб. и доп. - М.: ИНФРА-М, 2004. 384 с.

8. Экономика предприятия: Учебник для вузов / Под ред. проф. В.Я. Горфинкеля, профр. В.А. Швандара / 4-е изд., перераб. и доп. - М.: ЮНИТИ-ДАНА, 2007. 670 c.

кузНЕцОв Александр Павлович -

доктор технических наук, профессор кафедры станков МГТУ «СТАНКИН» 

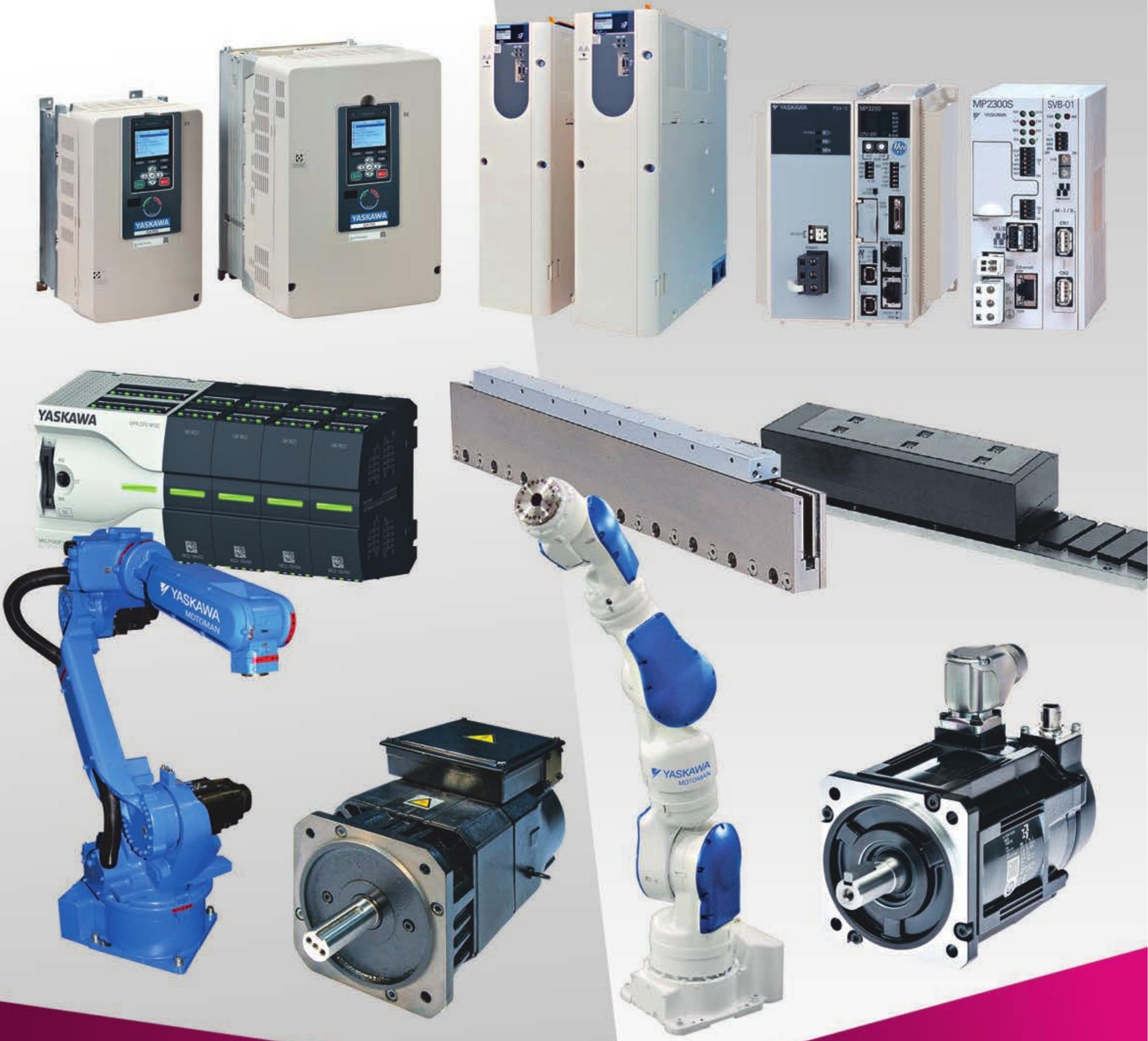
- Преобразователи частоты от 0,4 до 12000 кВт
- Шпиндельные двигатели
- Комплектные сервоприводы от 0,003 до 75 кВт
- Линейные сервосистемы
- Контроллеры управления движения
- Программируемые логические контроллеры
- роботы

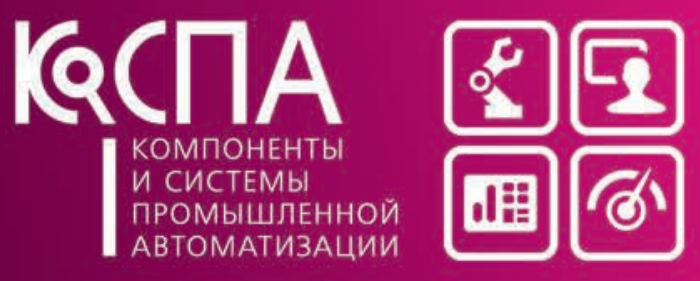

+7 (495) 660-28-22; www.cospa.ru

ООО «КОСПА» ОФИЦИАЛЬНЫЙ ПАРТНЕР И СЕРВИСНЫЙ ЦЕНТР YАSКАWА 